\title{
Dual- Energy CT (DECT) Pulmonary Angiography in Acute Pulmonary Thromboembolism (PTE): Causes, Semiology and Potential Diagnostic Pitfalls
}

\author{
Gálvez García ${ }^{*}{ }^{1}$, Muñoz Ruíz $\mathrm{AL}^{2}$, Nava Tomás $\mathrm{E}^{1}$, Chung $\mathrm{H}^{2}$ and Prieto Fernández $\mathrm{A}^{1}$ \\ ${ }^{1}$ Department of Radiology, Central University Hospital of Asturias (HUCA), Oviedo, Spain \\ ${ }^{2}$ Department of Radiology, Ronald Reagan- UCLA Medical Center, Los Ángeles, USA
}

${ }^{*}$ Corresponding author: Gálvez García S, MD., Department of Radiology, Central University Hospital of Asturias (HUCA), Oviedo, Spain, E-mail: saragalgar_87@hotmail.com

Citation: Gálvez García S, Muñoz Ruíz AL, Nava Tomás E, Chung H, Prieto Fernández A (2016) DualEnergy CT (DECT) Pulmonary Angiography in Acute Pulmonary Thromboembolism (PTE): Causes, Semiology and Potential Diagnostic Pitfalls. J Adv Radiol Med Image 1(2): 204. doi: 10.15744/24565504.1.204

Received Date: July 04, 2016 Accepted Date: October 25, 2016 Published Date: October 27, 2016

\begin{abstract}
The dual-energy computed tomographic (DECT) technique allows the differentiation of materials with large atomic numbers such as iodine. The basic principle of dual-energy CT is material decomposition based on attenuation differences at different energy levels. The parameters for iodine extraction are a minimum value of - $960 \mathrm{HU}$, and a maximum of $-600 \mathrm{HU}$. Lung parenchyma with normal perfusion in this attenuation range is presented in orange, and an area of decreased perfusion is visualized as an iodine defect area.

By using dual-energy CT angiography for the evaluation of perfusion defects in cases of pulmonary embolism (PTE), it is possible to replace perfusion and ventilation scanning. An iodine map from dual-energy CT can demonstrate the distribution of pulmonary perfusion. In patients with occlusive pulmonary emboli, iodine perfusion maps show wedge- shaped and in a segmental or lobar distribution iodine defect areas corresponding to malperfused lung parenchyma distal to a pulmonary embolus.

Therefore DECT pulmonary angiography not only enables imaging of emboli in the pulmonary vasculature but also provides an indication of their functional impact in terms of parenchymal perfusion.

The purpose of this study is to describe the use of DECT pulmonary angiography in acute PTE, analyzing the technique, clinical application and causes of false results in pulmonary parenchymal iodine perfusion maps.

Keywords: Dual energy; Computed tomography CT; Angiography Perfusion; Pulmonary embolism

List of Abbreviations: PTE: Pulmonary Thromboembolism; DECT: Dual- Energy CT; PBV: Pulmonary Blood Volume; PH: Pulmonary Hypertension
\end{abstract}

\section{Learning Objectives}

The purpose of our educational exhibit is to:

1. Present techniques, clinical applications, drawbacks and pitfalls of DECT in the diagnosis, prognosis and follow-up of PE.

2. Describe how to interpret iodine maps from dual-energy CT in cases of pulmonary embolism.

3. Evaluate the typical location and characteristics of focal iodine defects that are found in embolic and nonembolic patients.

4. Evaluate the impact of Pulmonary Blood Volume (PBV) maps on the detection of small pulmonary embolism.

5. List the clinical applications of dual-energy CT in various pulmonary diseases.

\section{Introduction and Technical Background}

Pulmonary embolism is a potentially fatal disorder and the third most common acute cardiovascular disease, after myocardial infarction and stroke. When properly diagnosed and treated, however, the associated mortality and recurrence can be reduced.

Pulmonary CT angiography has now largely replaced ventilation/ perfusion scintigraphy and conventional pulmonary angiography for the evaluation of possible PE. Unfortunately, conventional pulmonary CT angiography only provides morphological information and its ability to assess sub-segmental pulmonary arteries is variable $[1,2]$. 
Dual- energy CT technique provides a short scan time, superior anatomic detail and detects comorbid diseases, with a high detection rate of pulmonary embolism.

Dual-source CT uses two orthogonally mounted X-ray tubes and detectors to double the speed of image acquisition. As each tube can be set at a different tube potential ( $100 \mathrm{kVp}$ for one tube and $140 \mathrm{kVp}$ for the other), DSCT also allows for simultaneous dualenergy CT image acquisition and largely excludes the need for interscan changes in contrast enhancement or patient movement between CT acquisitions.

DECT data can be readily post-processed to determine the amount of iodinated contrast material in any voxel. This is because iodine, as compared with soft tissue, attenuates $\mathrm{X}$ - ray spectra very differently at $100 \mathrm{kVp}$ and $140 \mathrm{kVp}$ settings. The image data from both energy levels are combined to form a hybrid image that is equivalent to a scan acquired at $120 \mathrm{kVp}$, which can be used for diagnostic evaluation of morphology and anatomy. In combination with the iodine map, which is related to the microvascular circulation in the lung, this scan can provide morphological and functional information in terms of parenchymal perfusion, thus improving the CT assessment of subsegmental pulmonary arteries [2].

Dual energy CT explores the different attenuation properties of matter at the two energy levels (100KvP and $140 \mathrm{KvP})$. A lower $\mathrm{KvP}$ is associated with increase photoelectric interactions especially in substances with higher atomic number such as iodine and calcium. The attenuation value at two different energies within a voxel can subsequently be analyzed using material decomposition technique, and post-processing of images can provide additional diagnostic value compared to single energy conventional CT.

Iodine, as compared with soft tissue, attenuates X-ray spectra very differently at $100 \mathrm{kVp}$ and $140 \mathrm{kVp}$ settings. The image data from both energy levels are linearly blended to form a hybrid image, which can be used for diagnostic evaluation of morphology and anatomy [2].

The normal lung shows homogeneous distribution of iodine in the entire parenchyma, whereas in patients with occlusive pulmonary emboli, iodine perfusions maps show sharply delineated triangular- shaped subpleural iodine defect areas corresponding to embolism [1] (Figure 1).

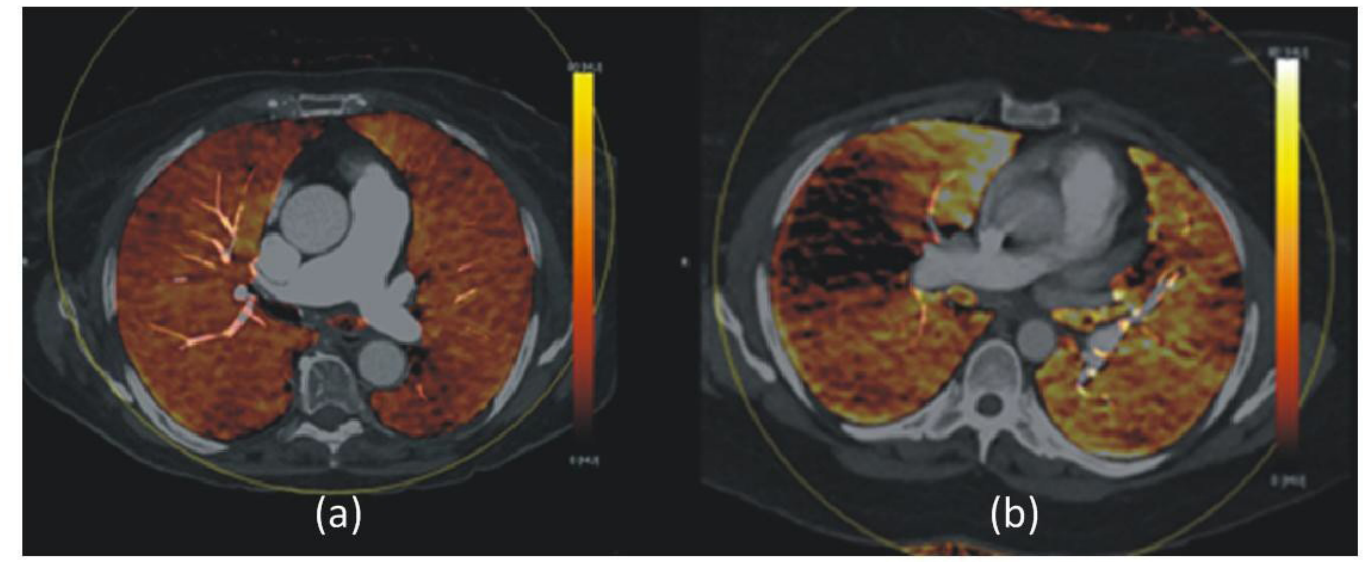

Figure 1: Normal parenchymal perfusion vs parenchymal perfusion defect due to $\mathrm{PE}$

(a) Axial dual- energy CT image shows normal parenchymal perfusion that manifests as homogeneous distribution of color between pulmonary vasculature.

(b) Axial dual- energy CT image shows a parenchymal perfusion defect due to an occlusive thrombus already seen in the CT pulmonary angiography.

References: Radiology, Central University Hospital of Asturias (HUCA), Oviedo- Spain.

With these advances new pitfalls are also introduced. There are many other causes of focal iodine defects which do not originate from pulmonary embolism that must be considered and may be distinguished by their lack of a wedge shape $[1,2]$.

The causes of nonembolic focal iodine defects are mostly due to beam- hardening artifacts from non-mixed contrast media in the superior vena cava or the left innominate vein for the apices of both the upper lobes, cardiac motion especially in the middle lung area, and diaphragmatic motion in the lung bases [1].

Areas of emphysema, atelectasis, or consolidation and lung masses are demonstrated also as perfusion defects. With an understanding of the common locations, characteristics, and causes of non-true-embolic defects, radiologists should be able to differentiate them from true- embolic defects.

\section{Acquisition of Pulmonary CT Angiography}

All CT examinations were performed in our hospital using a dual- source CT scanner (Somatom Definition, Siemens Healthcare) in dual- energy mode. The system is equipped with two x-ray tubes and two corresponding detectors, which are oriented in the gantry with an angular offset of 90 degrees. Each tube is set at a different tube potential: $100 \mathrm{kVp}$ for one tube and $140 \mathrm{kVp}$ for the other. The first detector array (corresponding to tube A) provides a field of view (FOV) of $50 \mathrm{~cm}$; and the second detector array 
(corresponding to tube B) is restricted to an FOV of $26 \mathrm{~cm}$ (Figure 18). Patients were centrally placed in the scanner to ensure that the entire pulmonary parenchyma was covered by the smaller field-of-view of the second tube detector array. In patients with a large body habitus, peripheral lesions can be excluded because data are only acquired by the second tube detector inside this area; thus, dual- energy data manipulation can only be performed in this area [3-5].

They were obtained after the injection of a bolus of iodine contrast followed by a $40 \mathrm{ml}$ saline flush via an 18- gauge catheter at $4 \mathrm{ml} \mathrm{s}-1$ into the antecubital vein. For automatic bolus triggering, a region of interest (ROI) was placed at the ascending aorta, and when the ROI reached the trigger threshold (65HU), the CT scan was performed [1].

DECT pulmonary angiography does not expose patients to any significant additional radiation exposure over that of standard CT pulmonary angiography $[3,4,6]$.

\section{Reconstruction of Pulmonary CT Angiography}

The acquired images are then automatically reconstructed to three separate image sets: $100 \mathrm{kVp}, 140 \mathrm{kVp}$ and a mixed 100:140 $\mathrm{kVp}$ image with weighting factor of 0.4 (40\% image information from the $100 \mathrm{kVp}$ image and $60 \%$ information from the $140 \mathrm{kVp}$ image).

Iodine perfusion CT scans are generated using the lung Perfused Blood Volume (PBV) application mode of dedicated dualenergy post processing software (Syngo.via, Siemens Medical Solutions). This application mode was designed for iodine mapping to generate blood flow imaging that reveals indirect conclusion of the blood flow parameters by using known attenuation range values for air, soft tissue and iodine at $100 \mathrm{kVp}$ and $140 \mathrm{kVp}$ to calculate their relative contributions to the voxel. Using this software various applications modes of material decompositions are available (PBV, Iodine Perfusion and Virtual Non- enhancement) [1].

PBV maps can be fused as an overlay on weighted- average images, which permits simultaneous gray - scale evaluation of the enhanced central vasculature and color-coded PBV evaluation of lung parenchymal enhancement (Figure 2).

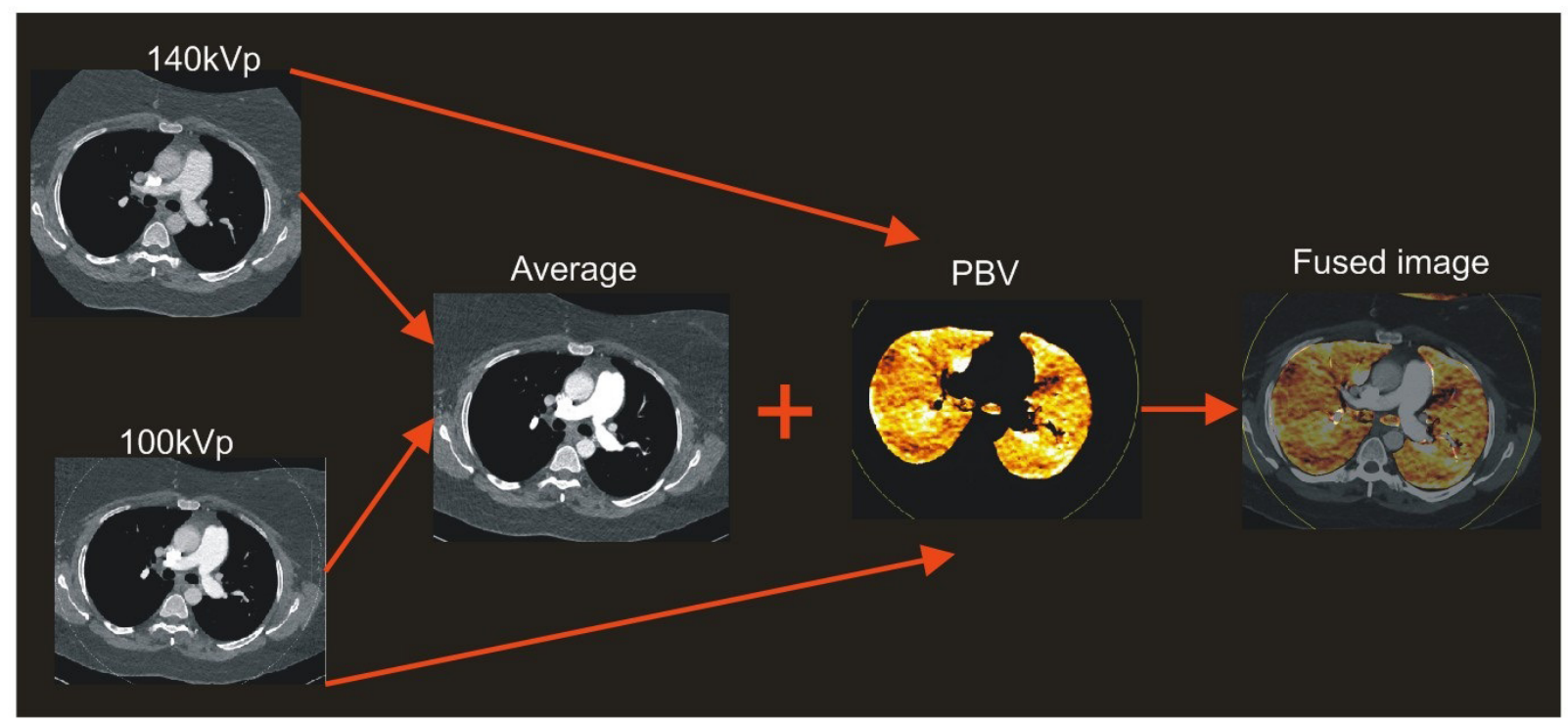

Figure 2: DECT PBV map generation

DECT PBV map generation. A PBV map is generated from $100 \mathrm{kVp}$ and $140 \mathrm{kVp}$ images using an algorithm that applies known attenuation- range values for air, soft tissue and iodine to calculate their relative contribution to each voxel. A Hounsfield- unit thresholding function is used to exclude the central vessels, allowing the PBV map to be overlaid on a weighted-average image to produce a fused PBV image. This enables simultaneous evaluation of the gray- scale vasculature with the color- coded PBV image representing parenchymal perfusion.

References: Radiology, Central University Hospital of Asturias (HUCA), Oviedo- Spain.

To generate an iodine perfusion map in PBV application mode, material decomposition can be performed only in the range between $-960 \mathrm{HU}$ and $-600 \mathrm{HU}$. Any areas with attenuation outside of this range are displayed as perfusion defects. Thus, areas of emphysema, atelectasis, or consolidation and lung masses are also demonstrated as perfusion defects. Therefore, the algorithm uses a thresholding function to define the volume of lung parenchyma for analysis, excluding the chest wall, mediastinum and central larger vessels [5].

In combination with the PBV map (iodine map), which is related to the microvascular circulation in the lung, this scan can provide morphological and functional information, thus improving the CT assessment of sub-segmental pulmonary arteries [2].

\section{Image Interpretation}

On the color- coded iodine perfusion (PBV) maps, lung parenchyma with normal perfusion is presented in orange. Focal iodine defects are presented as dark orange- or black- coloured areas [1]. 
PBV imaging can be used to identify contrast defects resulting from emboli. This tool is well established for depicting areas of hypoperfusion due to acute pulmonary embolism. However, not all pulmonary emboli are associated with PBV defects. Thus, the normal and abnormal appearance of PBV should be recognized.

Normal PBV images were defined as showing homogeneous contrast enhancement in the normal range (colour-coded orange) with dependent symmetric lung iodine distribution (Figure 3).
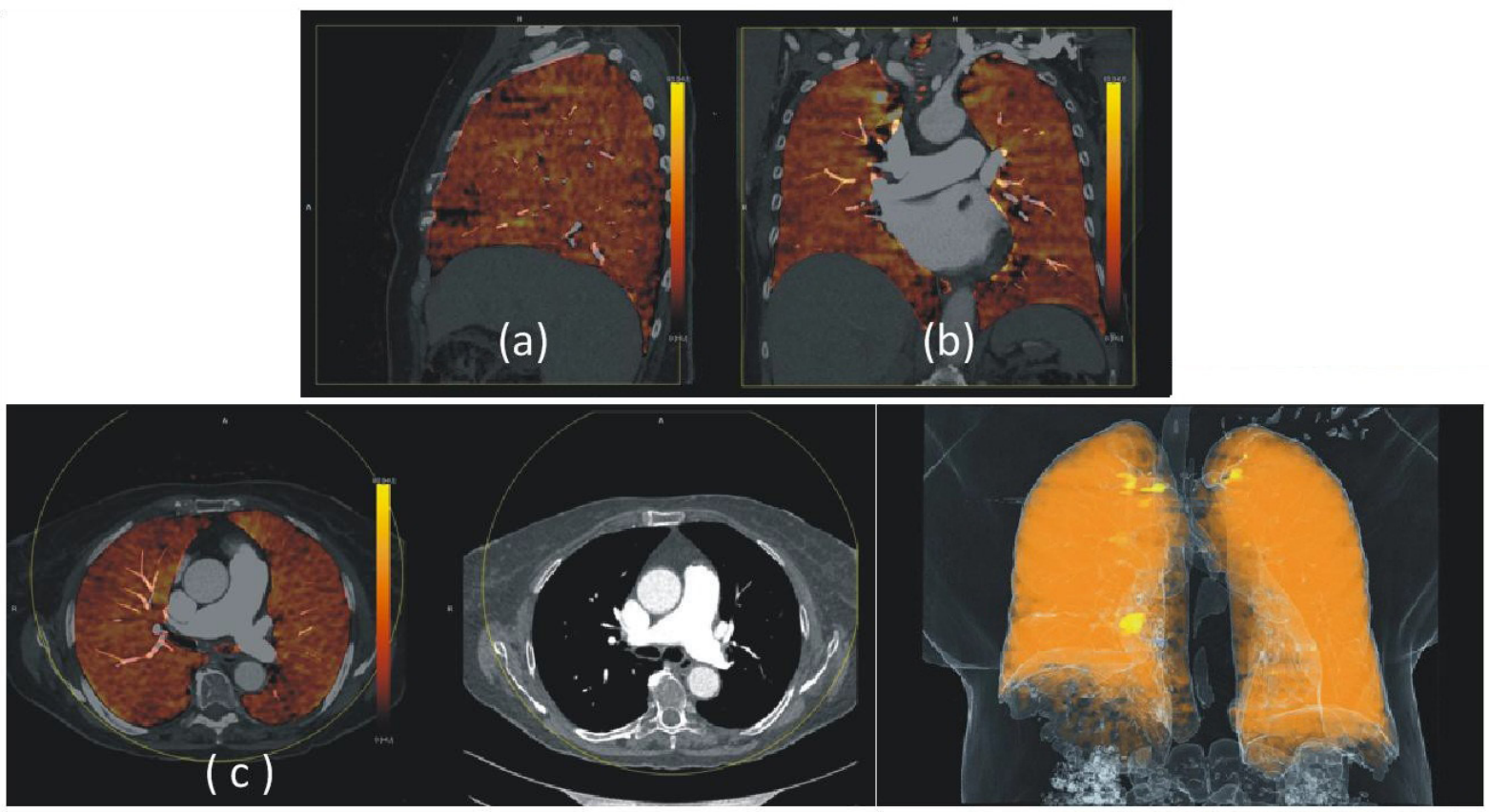

Figure 3: Normal appearance of pulmonary blood flow imaging

(a) Sagital, (b) coronal and (c) axial blood flow image. The images show homogeneous blood flow distribution in both lungs. Lung parenchyma with normal perfusion is seen as orange color.

References: Radiology, Central University Hospital of Asturias (HUCA), Oviedo- Spain.

Contrast enhancement defects that are consistent with PE include those that are peripherally located, wedge- sharped and in a segmental or lobar distribution (Figure 4,5,6 and 7).

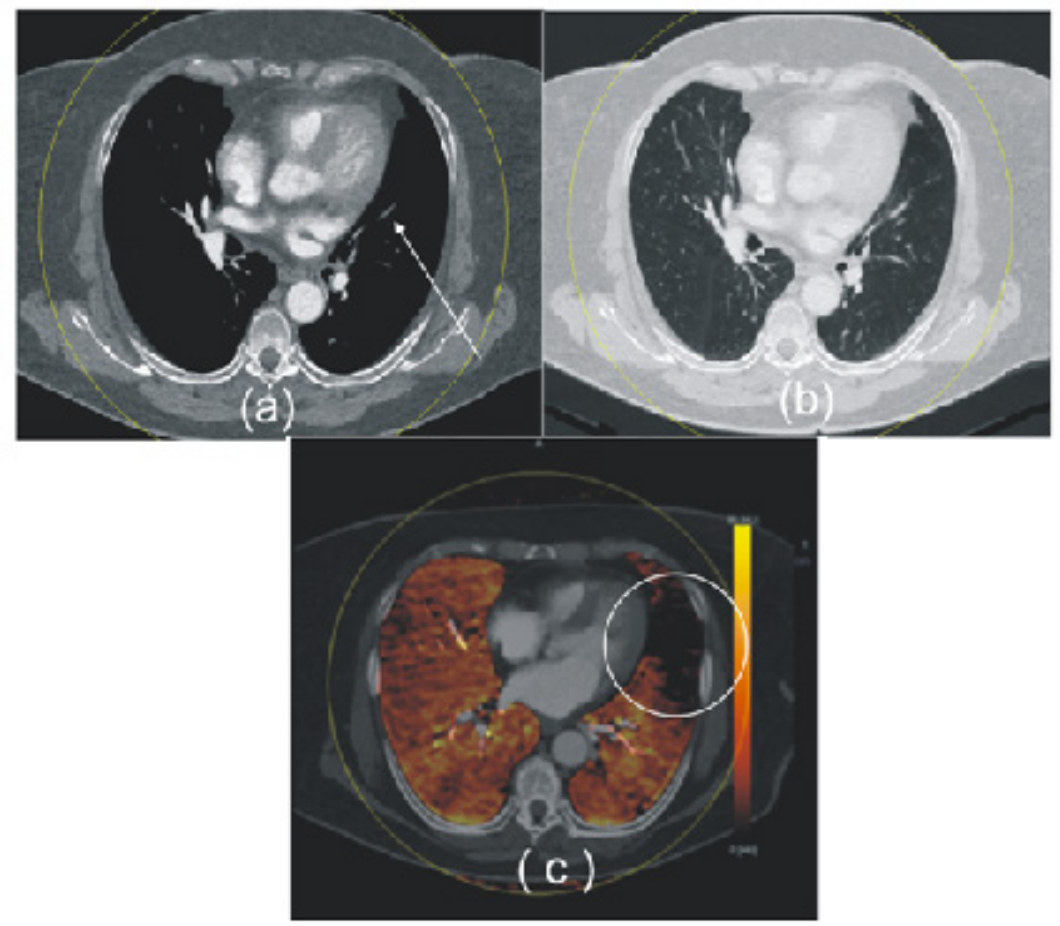

Figure 4: Acute pulmonary embolism

(a) Axial CT pulmonary angiography scan shows complete filing defect in pulmonary artery of lingular segment of left upper lobe (arrow)

(b) Normal lung parenchyma is seen on axial image CT viewed with lung window.

(c) Color- coded iodine perfusion map displays focal iodine defect in corresponding area of left upper lobe (circle).

References: Radiology, Central University Hospital of Asturias (HUCA), Oviedo- Spain. 


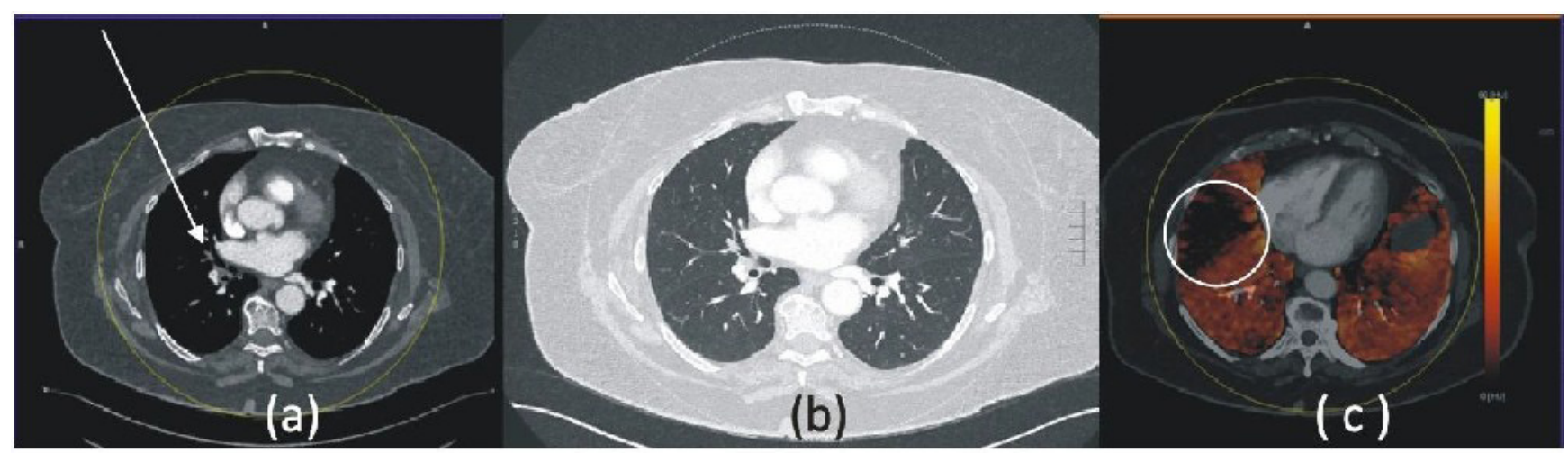

Figure 5: Acute pulmonary embolism

(a) An axial contrast- enhanced CT image shows occlusive thrombus within right middle lobe segmental pulmonary artery (arrow).

(b) Normal lung parenchyma is seen on axial image CT viewed with lung window.

(c) There is corresponding parenchymal perfusion defect in the axial blood flow image (white circle).

References: Radiology, Central University Hospital of Asturias (HUCA), Oviedo- Spain.

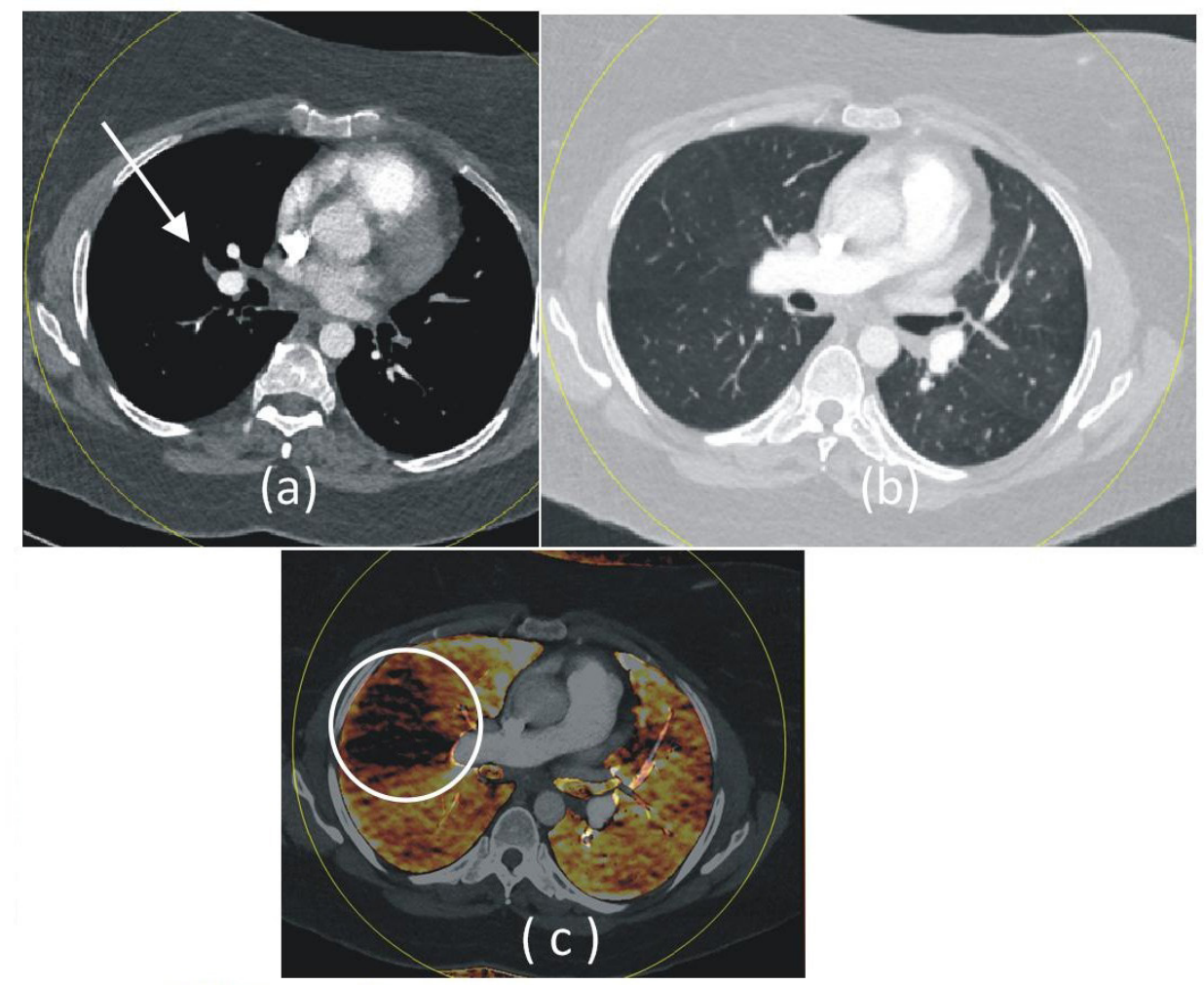

Figure 6: Acute pulmonary embolism

(a) An axial contrast- enhanced CT image shows occlusive thrombus within right middle lobe segmental pulmonary artery (arrow).

(b) Normal lung parenchyma is seen on axial image CT viewed with lung window.

(c) There is corresponding parenchymal perfusion defect in the axial blood flow image (white circle).

References: Radiology, Central University Hospital of Asturias (HUCA), Oviedo- Spain.

All other contrast enhancement defects, such as patchy to band- like defects without segmental distribution or complete loss of colour coding (indicating lack of air- containing voxels owing to consolidation), were inconsistent with PE in our experience.

DECT and PBV can increase the sensitivity of CTPA for tiny peripheral emboli (Figure 8) [4].

In routine clinical practice, small endoluminal clots in the segmental or subsegmental pulmonary arteries could not be detected at CT angiography. When an iodine map from dual- energy CT is used, the detectability of small endoluminal clots in segmental or subsegmental pulmonary arteries is expected to improve. Simultaneous evaluation of an iodine perfusion map is able to show perfusion defects; thus, detection performance can be improved [3].

The detection of small emboli is of clinical importance because even small emboli require treatment to prevent chronic PE and pulmonary artery hypertension $(\mathrm{PH})$ in several clinical scenarios: in patients with a small embolus and inadequate cardiopulmonary reserve; in patients who have a small embolus and coexisting acute deep venous thrombosis; and in patients with recurrent small emboli possibly owing to thrombophilia. The significance of small emboli needs to be studied further and PBV might assist with such studies [2]. 


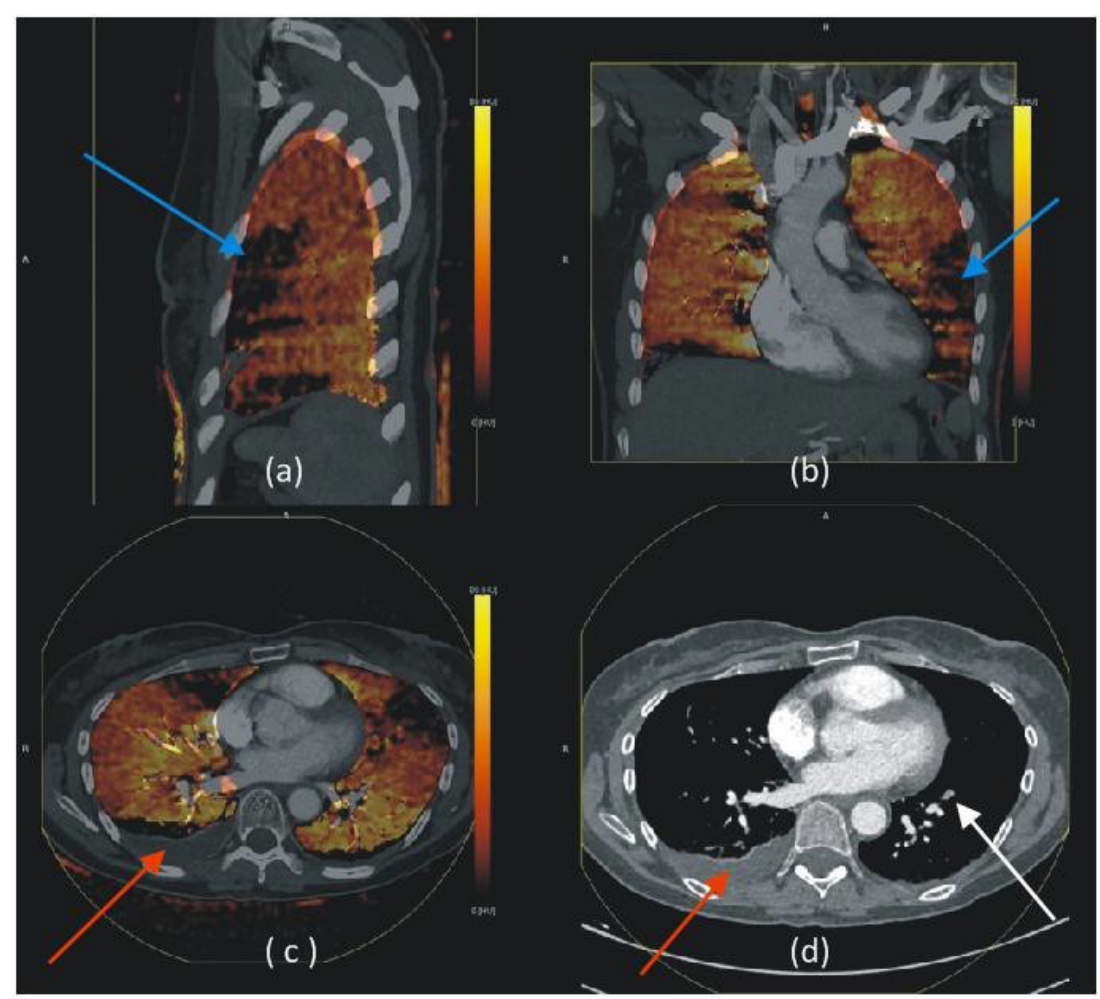

Figure 7: Acute pulmonary embolism and pleural effusion

(a) Sagital, (b) coronal and (c) axial blood flow images show a wedge-shaped contrast enhancement defect in the left lung inferior lobe. An axial contrast-enhanced CT image (d) shows a complete filling defect in pulmonary artery of left inferior lobe (white arrow), and also a right pleural effusion, that is seen in (c) as a focal iodine defect (red arrow). References: Radiology, Central University Hospital of Asturias (HUCA), Oviedo- Spain.
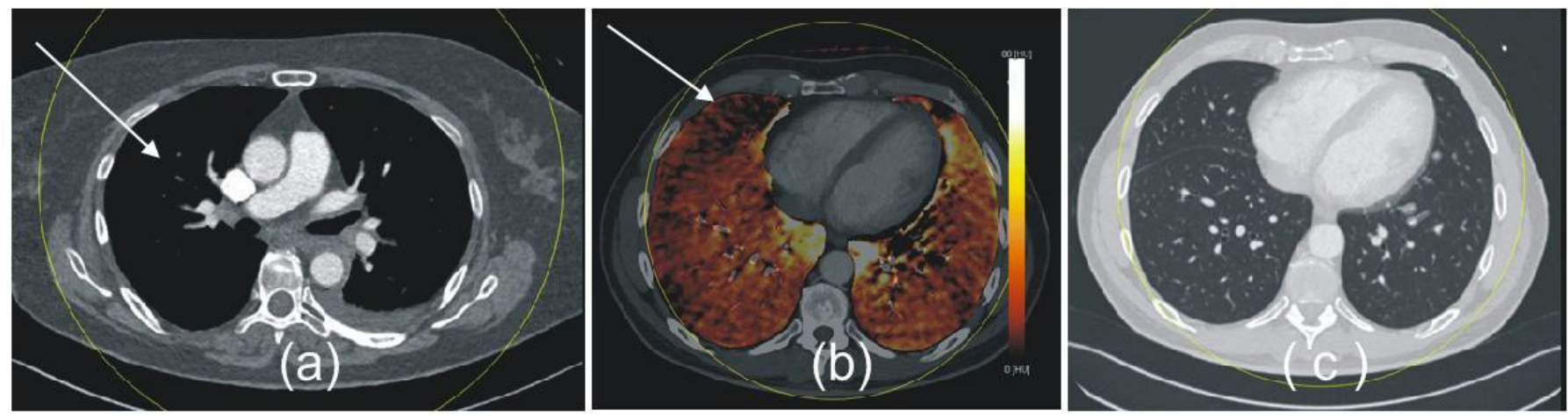

Figure 8: Peripheral emboli

(a) Axial CT image shows an endoluminal clot in the sub-segmental right middle pulmonary artery (arrow).

(b) Color- coded iodine perfusion map shows a focal sub-segmental filing defect (arrow) in the right middle lobe. In this case, the focal perfusion

defect helped in retrospective detection of the sub segmental clot, which was originally overlooked at axial CT.

(c) Normal lung parenchymal is seen on axial image CT viewed with lung window setting.

References: Radiology, Central University Hospital of Asturias (HUCA), Oviedo- Spain.

\section{Follow- up and chronic PE}

The iodine perfusion map may also be used for follow-up of pulmonary embolisms. After the patient receives anticoagulation therapy, we can observe a decrease in pulmonary perfusion defects as well as resolution of endoluminal clots [2].

DECT angiography not only depicts the contrast enhancement defects resulting from acute PE, but also shows contrast enhancement defects associated with chronic clots (Figure 9) [2,7,8].

\section{Pulmonary arterial hypertension $(\mathrm{PH})$}

Pulmonary arterial hypertension may be idiopathic or arise in association with chronic pulmonary thromboembolism. Chronic thromboembolic pulmonary hypertension affects approximately $4 \%$ of patients with acute pulmonary embolism. The CTPA findings of chronic thromboembolic pulmonary hypertension include parenchymal and vascular signs.

The characteristic morphologic CT features of chronic pulmonary arterial hypertension (PH) are dilatation of the pulmonary artery trunk, the diameter of which frequently exceeds that of the ascending aorta; dilatation of the right and left main pulmonary 
arteries; abrupt narrowing and tapering of the peripheral pulmonary vessels; right ventricular hypertrophy; and right ventricular and atrial enlargement with inversion of the interventricular septum and dilatation of the tricuspid valve annulus (Figure 10) [6-8].

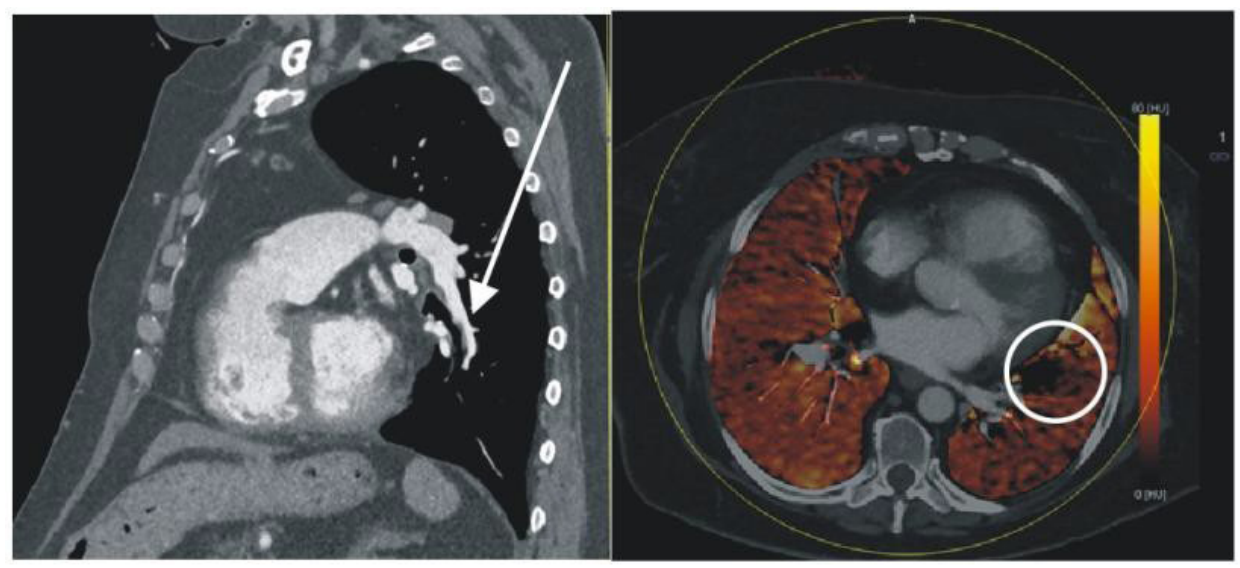

Figure 9: Chronic clots

(a) Sagital contrast- enhanced CT shows a mural embolus in the left lower lobe arter (arrow).

(b) The corresponding axial flow image shows a contrast enhancement defect in the left lower lobe.

References: Radiology, Central University Hospital of Asturias (HUCA), Oviedo- Spain.
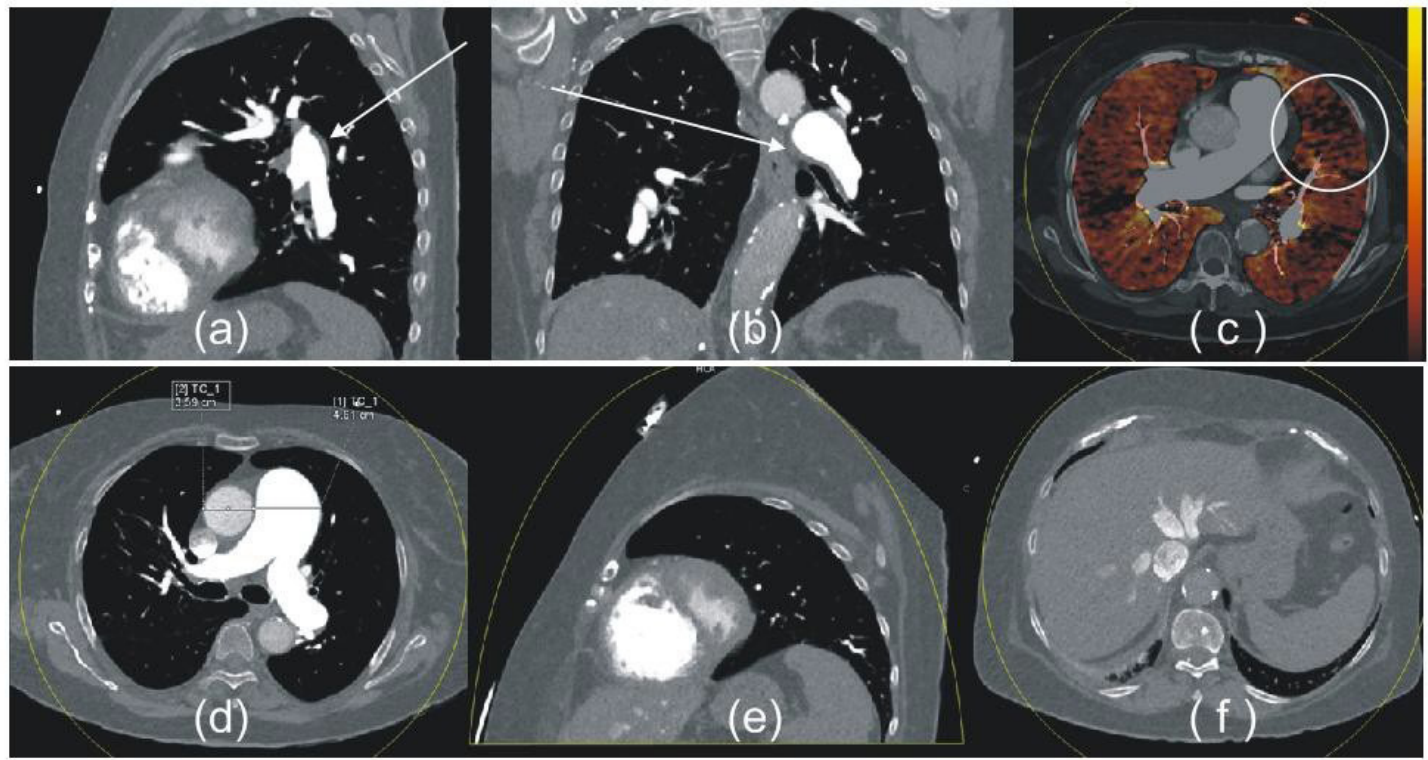

Figure 10: Vascular findings of chronic thromboembolic pulmonary hypertension. Patient with chronic thromboembolic pulmonary hypertension and right- sided heart failure

(a) Sagital and (b) coronal contrast-enhanced CT scan show wall-adherent thrombus (arrows) in the left pulmonary artery producing an irregular contour of the intimal surface. (c) Axial flow image shows pulmonary artery trunk dilatation and a contrast enhancement defect in the left lower lobe (circle).

(d) Axial CT image shows dilatation of the pulmonary artery trunk, the diameter of which frequently exceeds that of the ascending aorta. Right ventricular hypertrophy; and right ventricular and atrial enlargement with inversion of the interventricular septum is seen in (e).

(f) Axial contrast-enhanced CT scan at a lower level shows opacification of the inferior vena cava and suprahepatic veins because of retrograde flow of contrast material, which is often seen in patients with elevated right atrial and right ventricular pressures.

References: Radiology, Central University Hospital of Asturias (HUCA), Oviedo- Spain.

\section{Pitfalls of image interpretation}

In the analysis of PBV images, sources of false-positive results should be kept in mind to avoid misdiagnoses. These pitfalls when interpreting PBV images can relate to artifacts from contrast material, physiological distribution of the contrast material and pulmonary pathology.

Perfusion defects due to beam- hardening and cardiac and diaphragmatic motion artifacts: The common locations of these contrast enhancement defects inconsistent with PE, are the anterior and apical segments of the right upper lobe, apical segment of the left upper lobe, and medial segment of the right middle lobe.

These artifacts must be considered when an unexpected contrast enhancement defect is noted adjacent to an area of high contrast enhancement. Because nonembolic perfusion defects are usually found in a similar location and are associated with beamhardening artifacts or motion artifacts, we can differentiate them from perfusion defects originating from pulmonary embolisms. In patients without pulmonary embolism, the perfusion defects are bandlike or crescent-shaped rather than wedge-shaped [4]. 
Optimization of contrast medium injection parameters, including the use of a saline chase, can improve the image quality of DECT and increase diagnostic confidence in cases of pitfalls due to beam- hardening artifacts [2,5].

A potential method to improve image quality in the vicinity of the cardiac chambers to avoid cardiac motion artifacts might be to synchronise data acquisition with electrocardio-graphic tracing, a technological development currently exclusively available for myocardial "perfusion" analysis; however, such methodology could also potentially introduce stair-step or misregistration artifacts and requires further study [2].

Lung segments and characterization of focal iodine defects: In both upper lobes, the main cause of perfusion defects is beamhardening artifacts that result from nonmixed contrast material in the superior vena cava or left brachiocephalic vein. In this case, the perfusion defect may appear as multiple ill- defined or sharply defined band shapes. We also can find band-shaped beamhardening artifacts on the original CT scan in the mediastinal or lung window setting (Figure 11) [2].

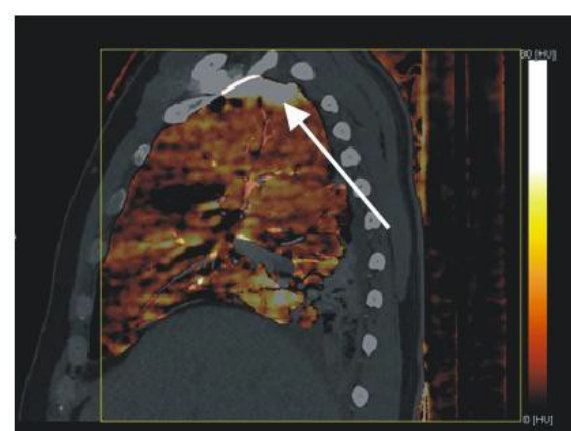

(a)

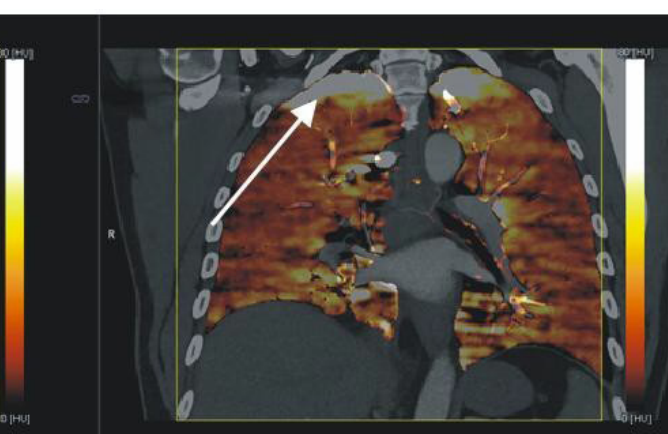

(b)

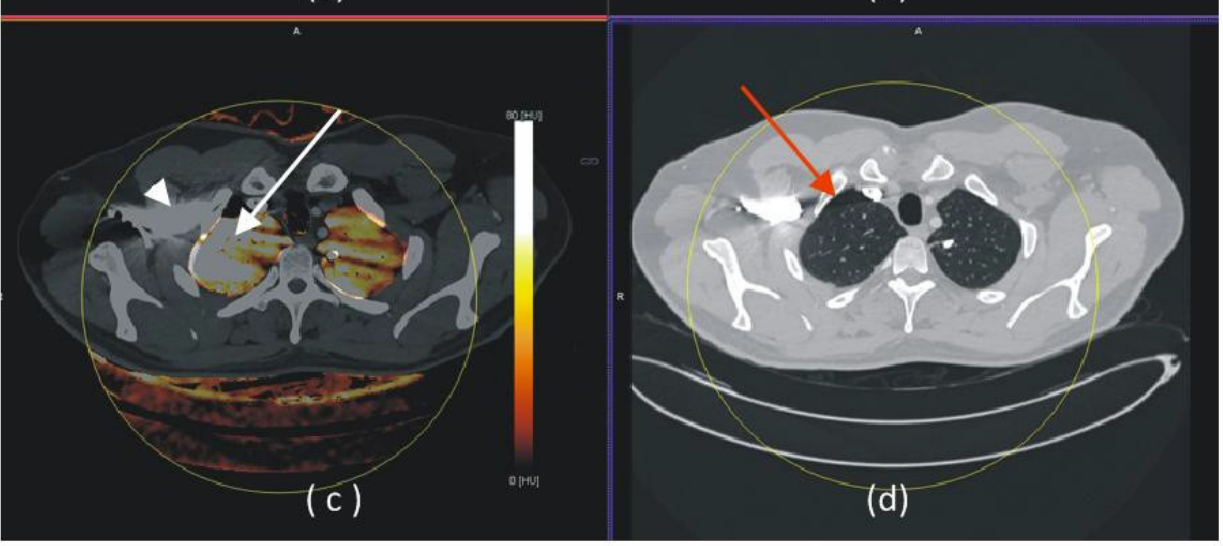

Figure 11: Beam-hardening artifact

(a) Sagital, (b) coronal and (c) axial color- coded perfusion map images show nonembolic band- shape perfusion defects in both upper lobe apices (white arrows). These defects are beam-hardening artifacts due to unmixed contrast material in the subclavian vein (arrowhead). (d) Lung window setting shows artifactual low attenuation band (red arrow) in both upper lobes. References: Radiology, Central University Hospital of Asturias (HUCA), Oviedo- Spain.

In the medial segment of the right middle lobe and the lingular segment of the left upper lobe (middle lung area), the main cause of perfusion defects is cardiac motion. Such defects are crescent-shaped with margins of focal iodine defects usually ill defined. In these cases, the mediastinal or lung window CT scans show blurring or a double contour, adjacent to the cardiac border, which is indicative of cardiac motion (Figure 12) [5,9].

Finally, in the lung base, the cause of perfusion defects is diaphragmatic motion. In this case, the perfusion defect is crescentshaped along the diaphragmatic contour. We can also identify a double contour or blurring on images obtained with lung or mediastinal windows (Figure 13) [5,9].

In addition, polygonal- shaped focal iodine defects adjacent to the aorta or pulmonary artery seem to be caused by the beating motion of these great vessels [1].

Normal physiological gravity- dependent variation in pulmonary "perfusion" should also be recognized [2].

Perfusion defects due to parenchymal diseases: Several lesions that cause increased parenchymal attenuation or profound decreased parenchymal attenuation can also manifest as perfusion defects. Those pseudodefects result from the thresholding function, which defines the range of included attenuations in the PBV calculation. Voxels with Hounsfield unit values outside of the range $(-960,-600 \mathrm{HU})$ are assigned a value of 0 and are displayed as black on the PBV image. These areas then mimic areas of genuine perfusion defects [9]. 


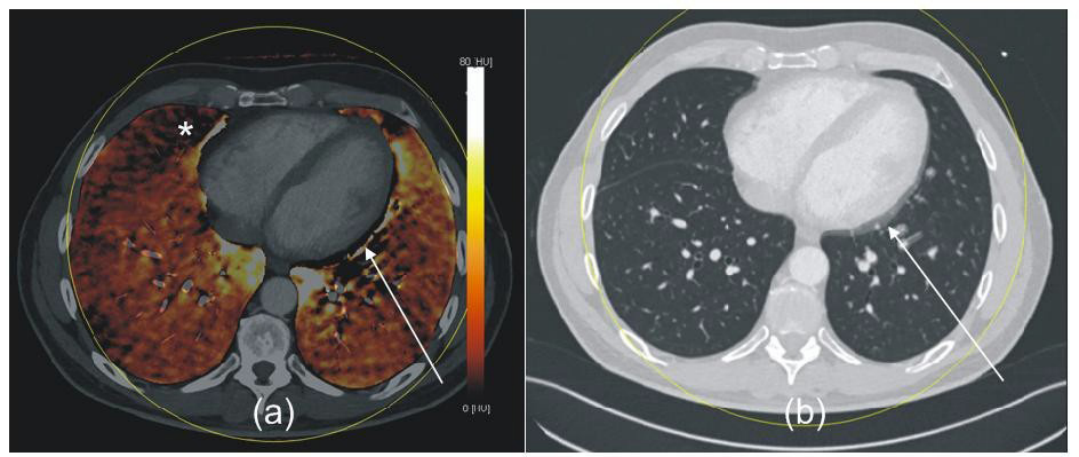

Figure 12: Patient without other CT evidence for pulmonary embolism

(a) Iodine perfusion map shows focal iodine defects in right middle lobe (asterisk) and lingular segment of left upper lobe (arrow) that are not associated with pulmonary embolism.

(b) Lung window setting shows double contour (arrow) along left cardiac border due to cardiac motion. References: Radiology, Central University Hospital of Asturias (HUCA), Oviedo- Spain.
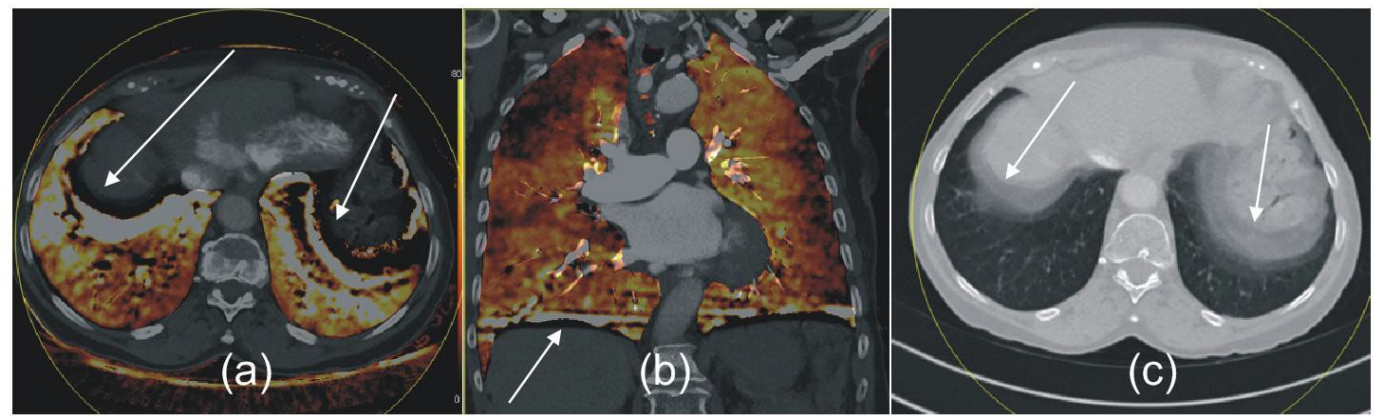

Figure 13: Diaphragmatic motion artifact

(a) Axial and (b) coronal iodine perfusion map images show focal iodine defect in base of both lower lobes (arrows). Focal iodine defect is crescent shaped and located along diaphragmatic contour.

(c) Lung window setting shows double contour (arrows) along diaphragm margin suggesting motion artifact due to diaphragmatic motion. References: Radiology, Central University Hospital of Asturias (HUCA), Oviedo- Spain.

These lesions are rarely confused with embolism because we are able to match it in the CT scan. A perfusion abnormality on blood flow imaging is not specific for PE, therefore functional reconstructions should not be viewed in isolation; rather, functional reconstructions should be viewed alongside the anatomic CT reconstructions for recognition of pulmonary abnormalities and diseases, such as emphysema, tumors invading or compressing the pulmonary arteries, pulmonary consolidation, vasculitides or aberrant vascular supply, all of which will result in blood pool defects in PBV images [9].

In the case of emphysema, the cause of perfusion defects is a true decrease in pulmonary circulation secondary to lung destruction; in the area of emphysema, alveolar surface reduction is accompanied by an equal reduction in capillary volume (Figure 14) [5].

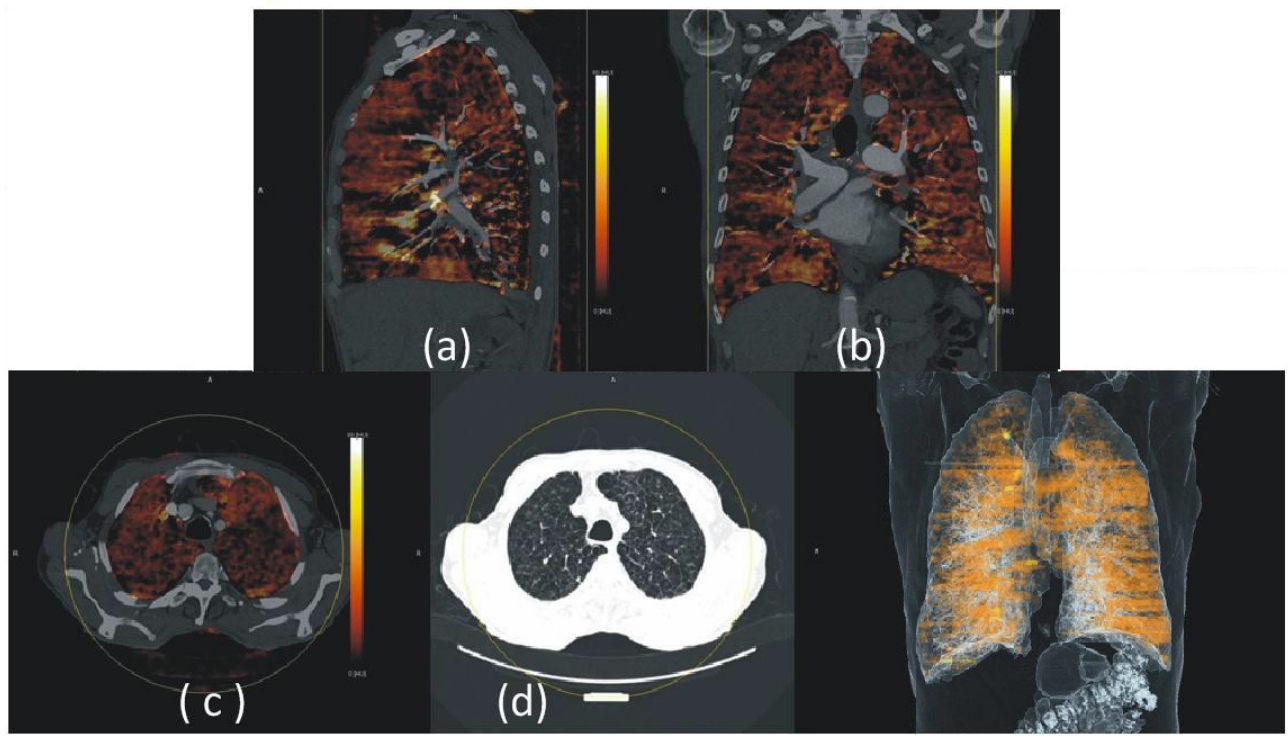

Figure 14: Contrast defect in the blood flow image caused by emphysema

(a) Sagital, (b) coronal, (c) and axial blood flow images show heterogeneous contrast enhancement in both lungs caused by the emphysema, readily seen on (d) axial image viewed with lung window. References: Radiology, Central University Hospital of Asturias (HUCA), Oviedo- Spain. 
In the case of lung masses (Figure 15,16), atelectasis (Figure 17), lung infarction, (Figure 18,19 and 20), consolidation, pneumonia (Figure 21), or pulmonary edema caused by congestive heart failure (Figure 22), the cause of perfusion defects is considered to be technical failure $[5,9]$.

In PBV application mode, to generate an iodine perfusion map, material decomposition could be performed only in the range between $-960 \mathrm{HU}$ and $-600 \mathrm{HU}$. Any areas with attenuation out of this range are displayed as perfusion defects.

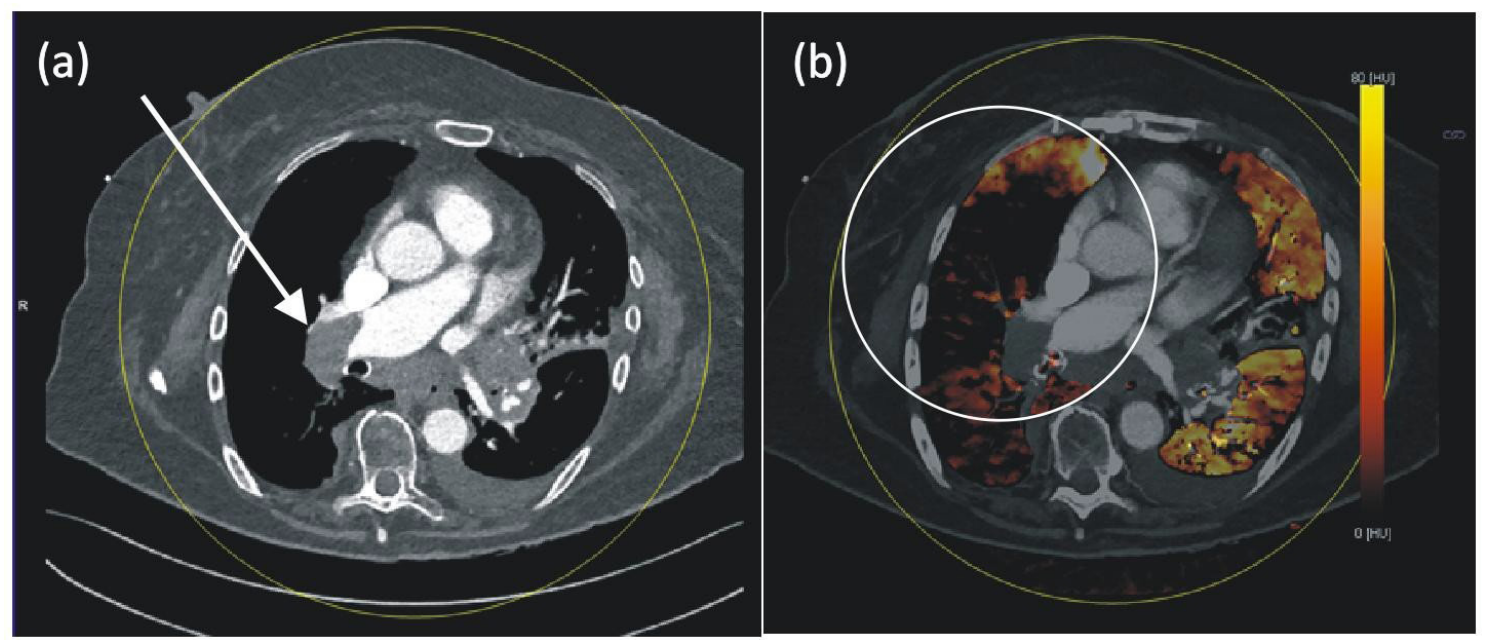

Figure 15: Contrast defect in the pulmonary blood volume image caused by lung carcinoma

(a) Axial image shows a right lung hiliar carcinoma invading the right pulmonary artery (arrow) resulting in diffuse decreased contrast enhancement on the right upper lung in the corresponding axial blood flow image (b). References: Radiology, Central University Hospital of Asturias (HUCA), Oviedo- Spain.

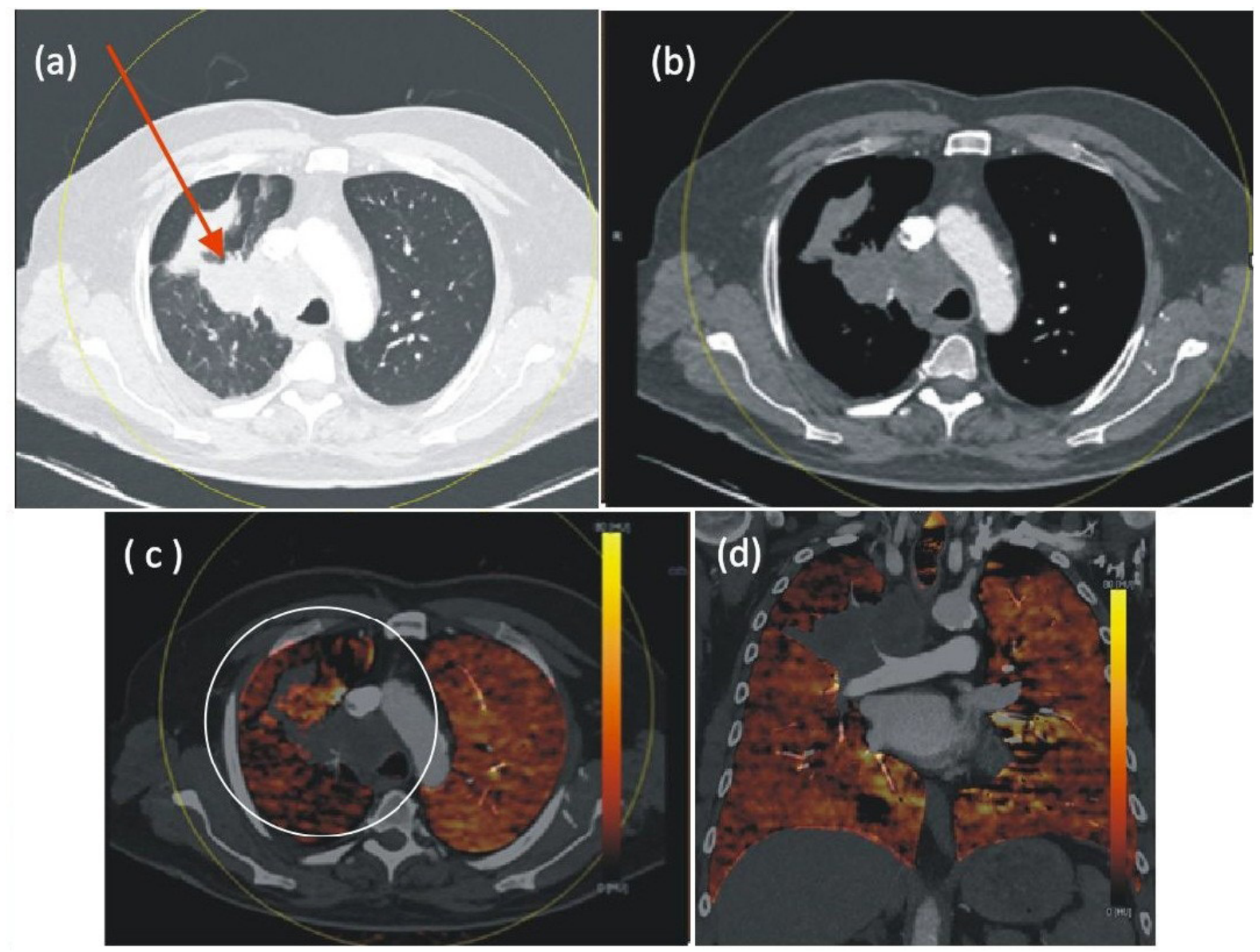

Figure 16: Contrast defect in the PBV caused by lung carcinoma

(a) And (b) axial CT angiographic images in lung and mediastinal window setting show right lung carcinoma (arrow) invading hiliar vasculature, resulting in diffuse decreased blood distribution within right upper lobe in pulmonary blood volume map (c) and (d). References: Radiology, Central University Hospital of Asturias (HUCA), Oviedo- Spain. 


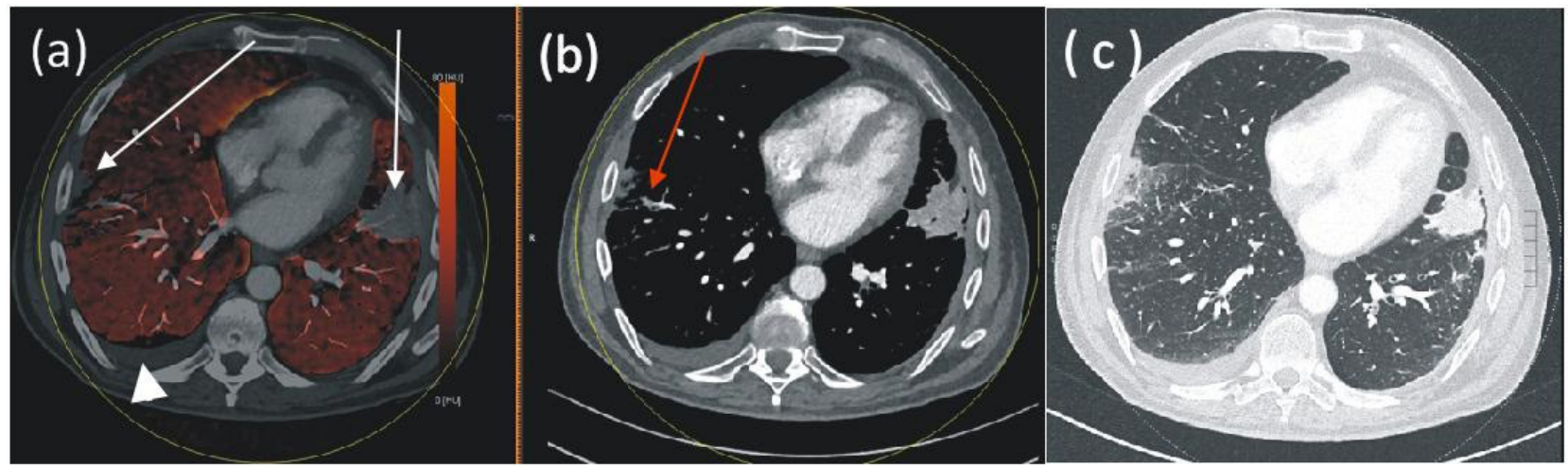

Figure 17: Contrast enhancement defects in the pulmonary blood flow image caused by lung consolidations- atelectasis and lung infarction Axial blood flow image (a) shows two contrast enhancement defects in the left lower lobe and in the right lower lobe (arrows).

The corresponding axial CT image in mediastinal (b) and lung (c) window setting clearly shows an atelectasis in the left lower lobe and a wedge shaped pleurally based opacification corresponding to a pulmonary infarction in the right lower lobe.

Axial contrast- enhanced CT image (b) also shows a corresponding occlusive filling defect representing emboli in the right lower lobe sub-segmental pulmonary artery (red arrow).

A small right pleural effusion is seen as a contrast enhancement defect as well in the blood flow axial image (a) (arrowhead).

References: Radiology, Central University Hospital of Asturias (HUCA), Oviedo- Spain.

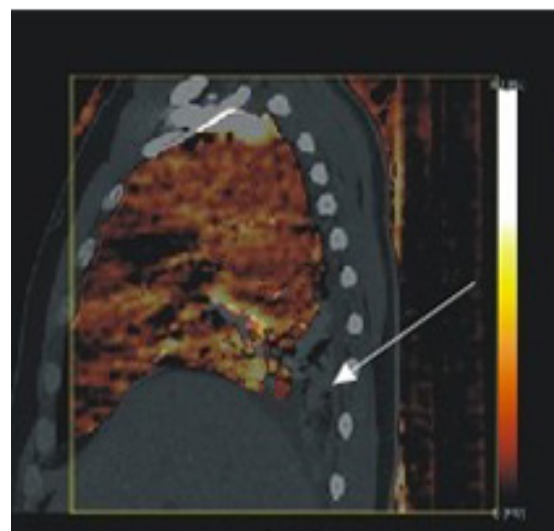

(a)
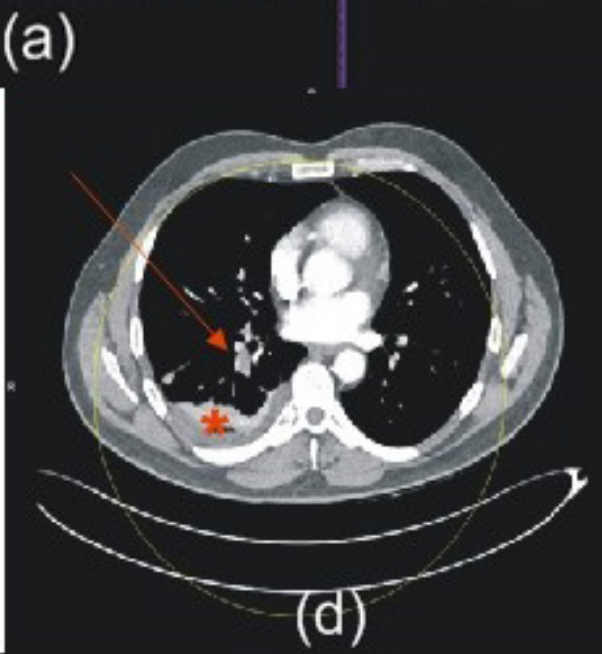

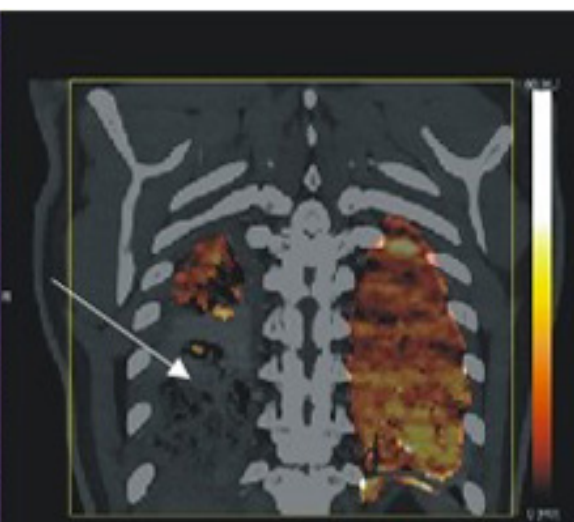

(b)
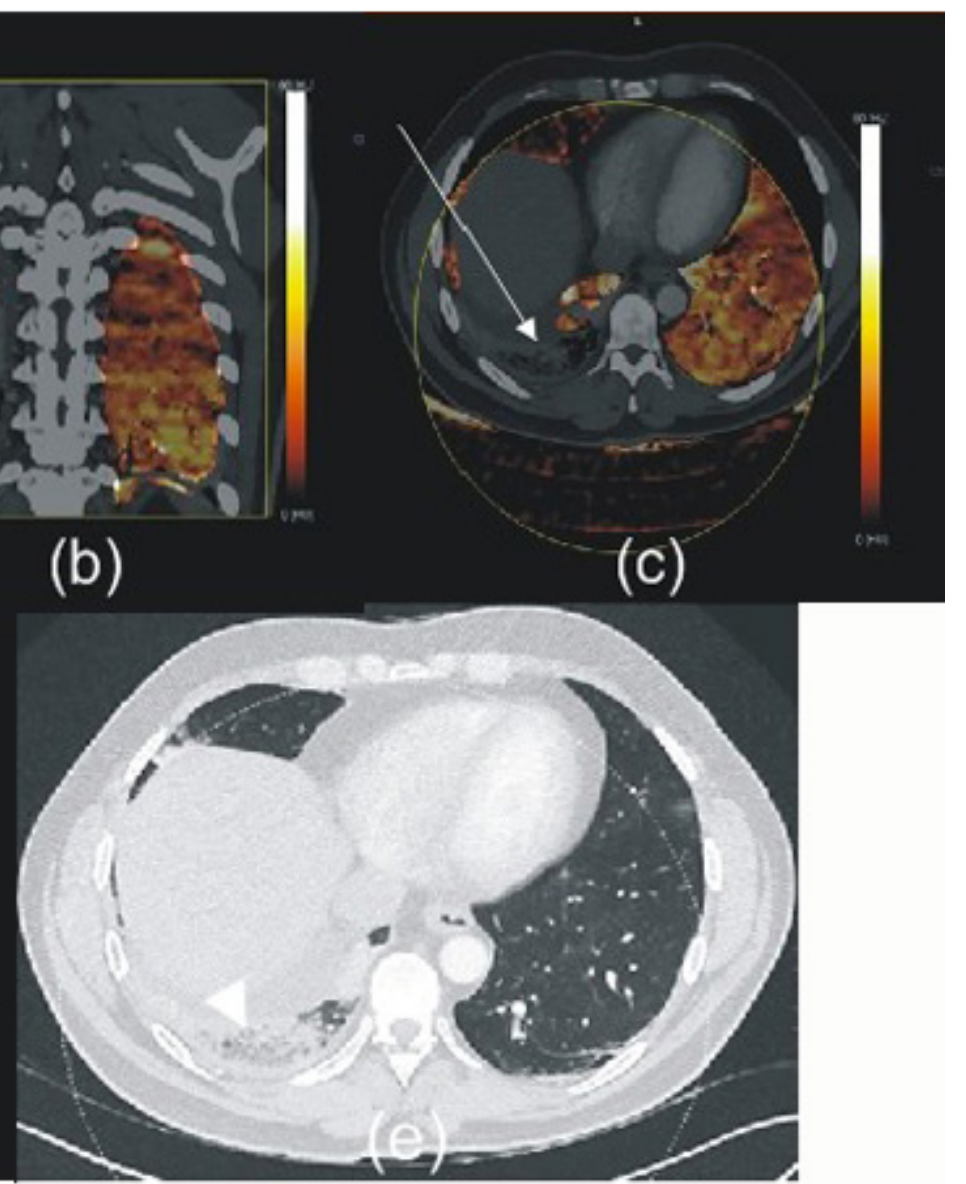

Figure 18: Focal iodine defect due to lung infarction

(a) Sagital, (b) coronal and (c) axial iodine perfusion image show a perfusion defect in the right lower lobe due to lung infarction (white arrow). (d) Axial CT angiography image shows a corresponding occlusive filling defect representing emboli in the right lower lobe segmental pulmonary artery (red arrow). (e) Axial CT lung window setting image shows the wedge shaped pleurally based opacification corresponding to the pulmonary infarction in the right lower lobe (arrowhead). To generate the iodine perfusion map, material decomposition was performed only in the range between - $960 \mathrm{HU}$ and $-600 \mathrm{HU}$. In the area of lung infarction the attenuation value is 50-60 HU; thus, material decomposition was not performed.

Note the right basal atelectasis $\left({ }^{*}\right)$.

Note in (c) yellow line marks boundary of dual-energy reconstruction field, due to the different fiel of view (FOV) of the two X- ray tubes of the DECT scan: detector A has a FOV of $50 \mathrm{~cm}$ and detector B has a restricted FOV of $26 \mathrm{~cm}$.

References: Radiology, Central University Hospital of Asturias (HUCA), Oviedo- Spain. 


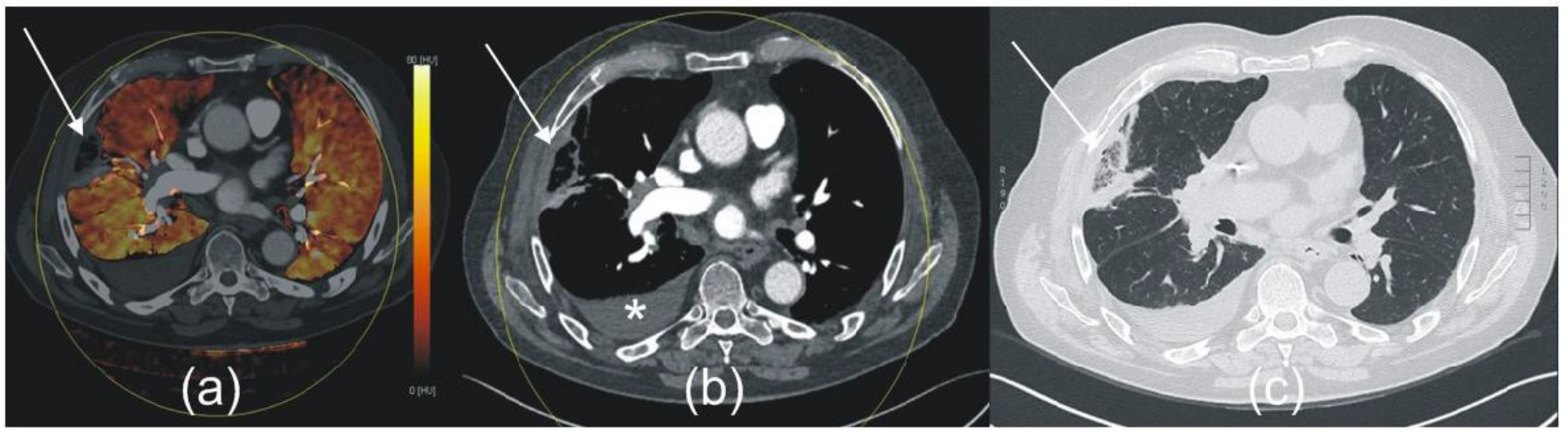

Figure 19: Focal iodine defect due to lung infarction

(a) Axial iodine perfusion image shows a perfusion defect in the right middle lobe due to lung infarction (arrow).

(b) Axial CT angiography image shows a corresponding occlusive filling defect representing emboli in the right middle lobe lateral segmental artery (arrow). (c) Axial CT lung window setting image shows the wedge shaped pleurally based opacification. This opacity is consistent with pulmonary infarction in the right middle lobe (arrow).

Note the right basal pleural effusion $(*)$.

References: Radiology, Central University Hospital of Asturias (HUCA), Oviedo- Spain.

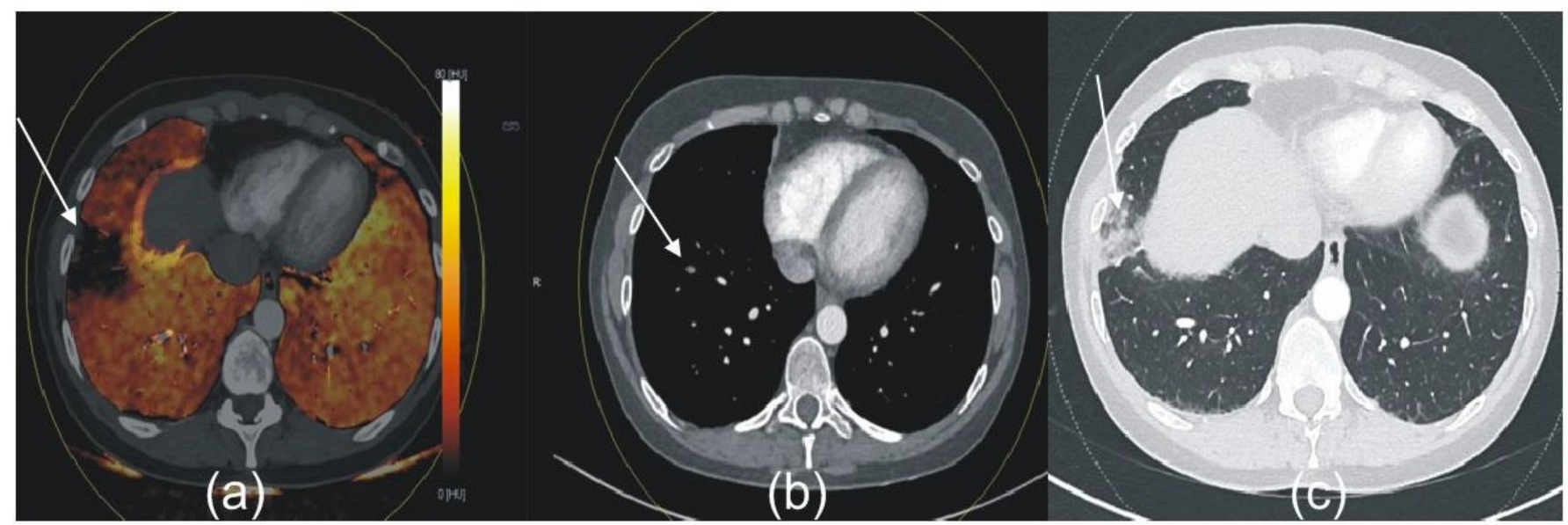

Figure 20: Lung infarction

(a) Axial iodine perfusion image shows a perfusion defect in the right lower lobe due to lung infarction (arrow).

(b) Axial CT angiography image shows a corresponding occlusive filling defect representing emboli in the right lower lobe segmental artery (arrow).

(c) Axial CT lung window setting image shows the wedge shaped pleurally based opacification consistent with pulmonary infarction in the right lower lobe (arrow). References: Radiology, Central University Hospital of Asturias (HUCA), Oviedo- Spain.

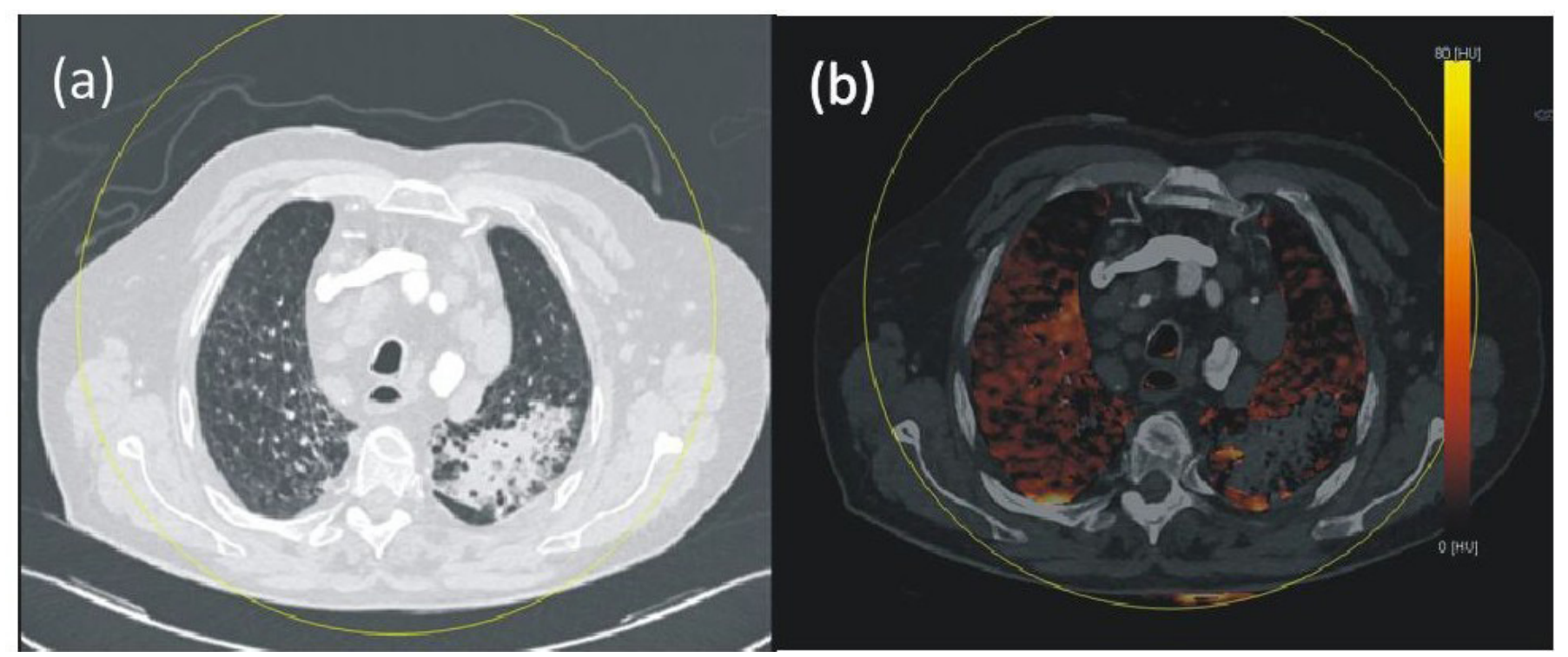

Figure 21: Patient with pneumonia in left upper lobe

(a) CT angiography scan image with lung window setting shows consolidation in the left upper lobe.

(b) Iodine perfusion map shows focal iodine defect in corresponding area of left upper lobe. Endoluminal clot was not delineated in any portion of pulmonary arteries on pulmonary CT angiography.

References: Radiology, Central University Hospital of Asturias (HUCA), Oviedo- Spain. 


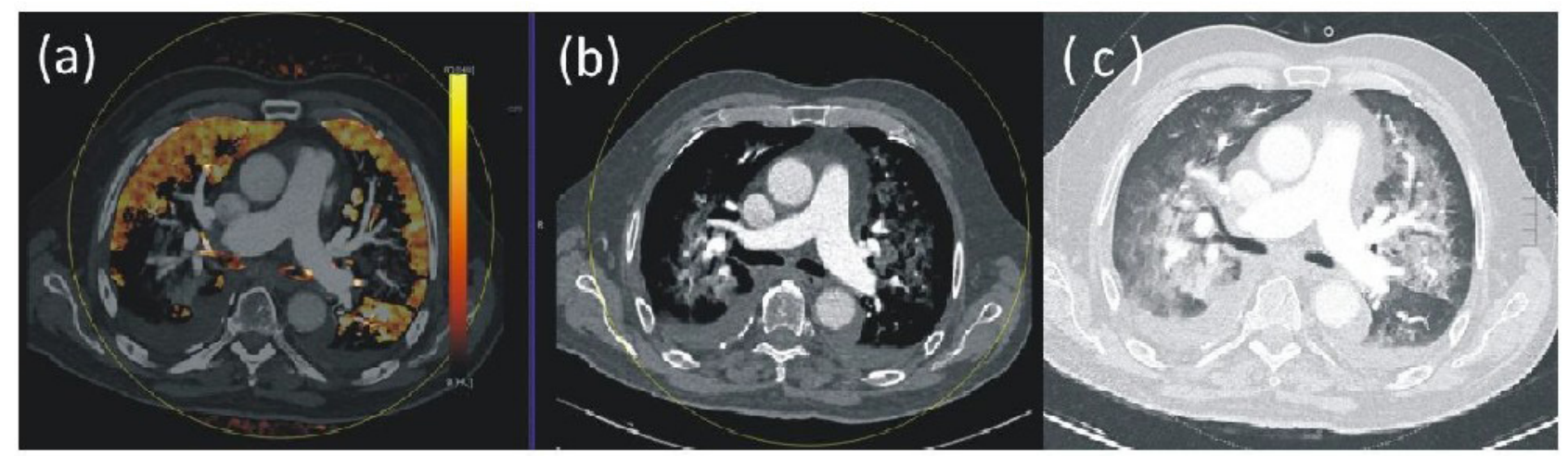

Figure 22: Patient with heart failure

(a) Axial pulmonary blood volume image shows bilateral areas of perfusion defects.

(b) Axial mediastinal and axial (c) lung window show bilateral dependent patchy areas of ground- glass attenuation and bilateral pleural effusion, which correspond to the areas of perfusion pseudo-defects seen in (a).

References: Radiology, Central University Hospital of Asturias (HUCA), Oviedo- Spain.

\section{Conclusion}

In conclusion, focal iodine defects not related to pulmonary embolism are frequently found in dual- energy pulmonary CT angiography scans.

Contrast enhancement defects that are consistent with PE include those that are peripherally located, wedge- shaped and in a segmental or lobar distribution. All other contrast enhancement defects, such as patchy or band- like defects without segmental distribution or complete loss of colour coding (indicating lack of air- containing voxels owing to consolidation), were inconsistent with PE [2,5].

The most common causes of nonembolic focal iodine defects are beam- hardening artifacts or artifacts due to motion. With an understanding of the common locations, characteristics and causes of non- true- embolic defects, we should be able to differentiate them from true- embolic defects [1].

Knowledge of the focal iodine defects not related to pulmonary embolism can help us to more accurately interpret dual- energy pulmonary CT angiography scans [1].

DECT enables the simultaneous assessment of vascular anatomy, parenchymal morphology, perfusion, and cardiopulmonary status with no extra radiation dose (the radiation dose for DECT is equivalent or less than conventional CT technique) [7]. Images of pulmonary parenchymal contrast enhancement obtained using DECT improve the detection of defects, increasing sensitivity for pulmonary emboli, in particular for small emboli at a subsegmental level or in more distal vessels. DECT also improves the diagnosis of other vascular disorders, lung malignancies and parenchymal diseases [2].

\section{References}

1. Kang MJ, Park CM, Lee CH, Goo JM, Lee HJ (2010) Focal iodine defects on color-coded iodine perfusion maps of dual-energy pulmonary CT angiography images: a potential diagnostic pitfall. AJR Am J Roentgenol 195: W325-30.

2. Lu GM, Wu SY, Yeh BM, Zhang LJ (2010) Dual-energy computed tomography in pulmonary embolism. Br J Radiol Aug 83: 707-18.

3. Sánchez-Gracián CD, Rodríguez CM, López CT (2011) Dual-energy computed tomography: what is it useful for? [La tomografía computarizada de doble energía: ¿para qué la quiero?]. Radiologia 10.1016/j.rx.2012.03.009.

4. Hoey ET, Gopalan D, Screaton NJ (2009) Dual-energy CT pulmonary angiography: A new horizon in the imaging of acute pulmonary thromboembolism. AJR Am J Roentgenol 192: W341-2.

5. Lu GM, Zhao Y, Zhang LJ, Schoepf UJ (2012) Dual- Energy CT of the Lung. AJR Am J Roentgenol 199: S40-53.

6. Ameli-Renani S, Rahman F, Nair A, Ramsay L, Bacon JL, et al. (2014) Dual-energy CT for imaging of pulmonary hypertension: challenges and opportunities. Radiographics 34: 1769-90.

7. Peña E, Dennie C, Veinot J, Muñiz SH (2012) Pulmonary hypertension: how the radiologist can help. Radiographics 32: 9-32.

8. Grosse C, Grosse A (2010) CT Findings in Diseases Associated with Pulmonary Hypertension: A Current Review. RadioGraphics 30: 1753-77.

9. Kang MJ, Park CM, Lee CH, Goo JM, Lee HJ (2009) Dual-energy CT: clinical applications in various pulmonary diseases. Radiographics 30: 685-98. 


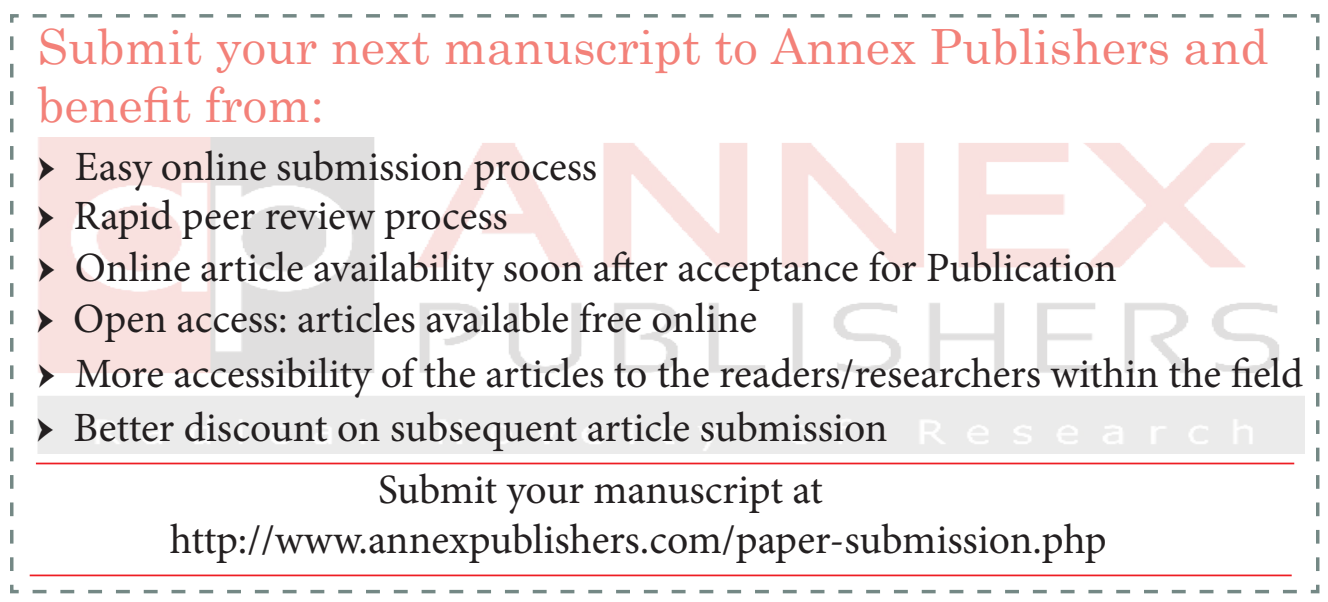

